\title{
TGP03
}

\section{Up-Going Drainhole Drilling for Reducing Liquid- Loading of Wells in Tight Gas Reservoir}

\author{
B. Li* (Baker Hughes Inc.), D. Sun (Baker Hughes Inc.), H. Grimmer (Baker \\ Hughes Inc.), D. Georgi (Baker Hughes Inc.), V. Krueger (Baker Hughes \\ Inc.), A. Jacques (TOTAL) \& J. Lassus-Dessus (TOTAL)
}

\section{SUMMARY}

Typically, tight gas (TG) reservoirs have very low permeability. Without stimulation, TG reservoirs cannot be produced economically. Hydraulic fracturing is an often-used efficient well stimulation technology, because it creates large contact areas through the reservoir. However, hydraulic fracturing is an uncontrollable technology.

Drainhole drilling is designed as an alternative stimulation technique. Drainholes may overcome the disadvantages of hydraulic fracturing. However, liquid-loading could be an issue for TG reservoir stimulated with down-going drainholes.

To overcome the liquid-loading problem, we propose to drill up-going drainholes. To verify whether this approach is valid, we performed a reservoir simulation study. The sensitivity analysis was performed for gas wells in a heterogeneous TG reservoir. From this simulation study, we conclude the following:

- Up-going drainholes can delay the occurring of the liquid-loading of wells in TG reservoirs. - For small kv/kh, the deviation angles of up-going drainholes need to be optimized to avoid or delay the liquid-loading. In addition one needs also to optimize length, phasing, and drilling density of drainholes. -The stimulation efficiency of a gas well located in a low permeability region is higher than that of a well located in a high permeability region. 
Typically, tight gas (TG) reservoirs have very low permeability $(<0.1 \mathrm{mD})$ and low porosity. Without stimulation, TG reservoirs cannot be produced economically. Hydraulic fracturing is an often-used efficient well stimulation technology, because it creates large contact areas through the reservoir. However, hydraulic fracturing is to some degree an uncontrollable, complex, and expensive technology.

Drainhole drilling is designed as an alternative stimulation technique. Drainholes can be thought of as long and ultra-thin boreholes emanating from a motherbore producing back to the surface. Drainholes may overcome the technical disadvantages of hydraulic fracturing, if the drainholes have sufficient length and drilling density. However, mobile waters may exist and initial water saturations may be very high in TG reservoirs. Also, the gas production rate in TG reservoirs typically declines rapidly, because of pressure depletion and extremely low permeability. Liquid-loading often is a severe issue for TG wells and could also be an issue for TG reservoir stimulated with down-going drainholes.

To overcome the liquid-loading problem, we propose to drill up-going drainholes. Theoretically, gas expansion energy at the top of the reservoir can be more efficiently utilized to produce gas by upgoing drainholes, compared with down-going drainholes. The bottom water zone can be avoided by the up-going drainholes. If the produced water in the up-going drainholes drains back to the motherbore the liquid removal will be simplified.

To verify whether this approach is valid and whether it will delay or mitigate the occurrence of liquidloading, we performed a reservoir simulation study using Turner's liquid-loading model. The sensitivity analysis was performed by varying the deviation angle of drainholes for gas wells in a heterogeneous TG reservoir. The gas and water production rates, WGR, bottom hole flowing pressures and wellhead pressures are monitored, and the well shut-in times are compared.

From this simulation study, we conclude the following:

- Up-going drainholes can delay the occurring of the liquid-loading of wells in TG reservoirs.

- For small $\mathrm{k}_{\mathrm{v}} / \mathrm{k}_{\mathrm{h}}$, the deviation angles of up-going drainholes need to be optimized to avoid or delay the liquid-loading. In addition one needs also to optimize the length, phasing, and drilling density of drainholes.

- Up-going drainhole drilling more effectively increases drainage areas of gas wells.

- The stimulation efficiency of a gas well located in a low permeability region is higher than that of a well located in a high permeability region.

- Gas wells located on sweet spots of TG reservoirs produce gas at higher rates. 